\title{
Correlation of Strain and Elemental Distribution at the Interface Using Simultaneous Energy-Filtered High-Speed 4D STEM Diffraction Imaging and EDS
}

\author{
Anahita Pakzad ${ }^{1}$ and Paolo Longo ${ }^{1}$ \\ 1. Gatan Inc., Pleasanton, CA, USA
}

In today's real-world materials science problems, usually the solution comes from not only one, but the combination of multiple types of experiments and various sources of information; in the high-speed competitive design and production of new electronic devices at the nanometer scale, the ability to accurately measure the mechanical properties, and spatially align and correlate data from multiple sources is imperative to form a clear picture of the interfacial phases present.

The scanning transmission electron microscope (STEM)-based diffraction imaging technique uses a parallel or convergent electron beam to generate a diffraction patterns at each scanning point. Using a high-speed CMOS camera, these two-dimensional diffraction patterns can be recorded for a twodimensional (2D) STEM rastered area, which results in a 4-dimensional (4D) STEM diffraction data cube; such 4D datasets can be processed to measure the shift in the diffraction spots (related to the crystal plane spacing) and generate strain maps of the scanned area. When the camera used for the collection of diffraction patterns is fitted at the end of an energy filter, the effect of plural scattering can be easily removed (increasing the pattern contrast and interpretability) by placing the energy selecting slit across the ZLP and allowing only the elastically scattered electrons to reach the detector. Energy dispersive $\mathrm{x}$ ray spectroscopy (EDS) is also a key technique for elemental composition analysis of materials, that has been used in STEM for several years. Although this technique does not provide any information on the chemical state of elements in the specimen, for interfacial analyses, EDS can be greatly useful when combined with other STEM techniques such as electron energy loss spectroscopy (EELS) or 4D STEM.

In this abstract, we present a very new approach where both energy-filtered 4D STEM and EDS data are collected simultaneously at high-speed. The combination of both techniques allows a more complete investigation across an interface region where structural changes can be coupled with the elemental distribution available using EDS. During such data acquisition approach, the high-speed direct detection CMOS camera (K2 IS, installed at the end of an energy filter) leads the acquisition as the master and provides synchronization by sending a pulse out at the end of each diffraction image acquisition. This pulse is then sent to the DigiScan unit to advance the beam to the next pixel position and to inform the EDS unit that the beam has moved. EDS, STEM and 4D STEM diffraction datasets are all acquired in DigitalMicrograph ${ }^{\mathrm{TM}}$ and can be stored on disk for future data analysis.

As an example of the advantages of combined 4D STEM and EDS data collection, the interface region between yttria-stabilized zirconia (YSZ) and $\mathrm{Fe}_{2} \mathrm{O}_{3}$ shown in Figure 1a was analyzed. Figures $1 \mathrm{~b}$-e show the EDS map of O K, Zr L and Fe K, the in- and out-of-plane strain maps, and the intensity profiles of these maps acquired across the selected region in Figure 1a. From the averaged strain curves, there appears to be strong variations in the local strain in proximity of the interface. The EDS maps show the interface to be quite abrupt although a careful observation of the $\mathrm{O}$ map shows some decrease of contrast right across the interface indicating a possible deficiency in oxygen. In order to obtain a better understanding of the elemental distribution across the interface, high-energy resolution core-loss EELS analysis was carried out. In this instance, the $\mathrm{Fe} \mathrm{L}_{2,3}$ data was acquired using the $\mathrm{K} 2$ camera in counting mode, leading 
to improved energy resolution due to the better point spread function, as well as higher spectral quality due to the reduced noise level, which is very important to resolve fine details in the near edge fine structure and extract chemical state information with improved sensitivity. Figure 2 shows the EELS spectra of Fe $\mathrm{L}_{2,3}$-edges extracted from the $\mathrm{Fe}_{2} \mathrm{O}_{3}$ film and near the interface. A significant change in the $\mathrm{Fe} \mathrm{L}_{2}$ and $\mathrm{L}_{3}$ ratio, as well as a chemical shift, are present, all these effects being consistent with changes in the valence state. The strain at the interface measured using 4D STEM could be the reason for such variations in the chemistry and composition across the interface [1].

\section{References:}

[1] We would like to thank Dr. Gianluigi Botton from McMaster University for providing the sample and helpful discussions on this work.
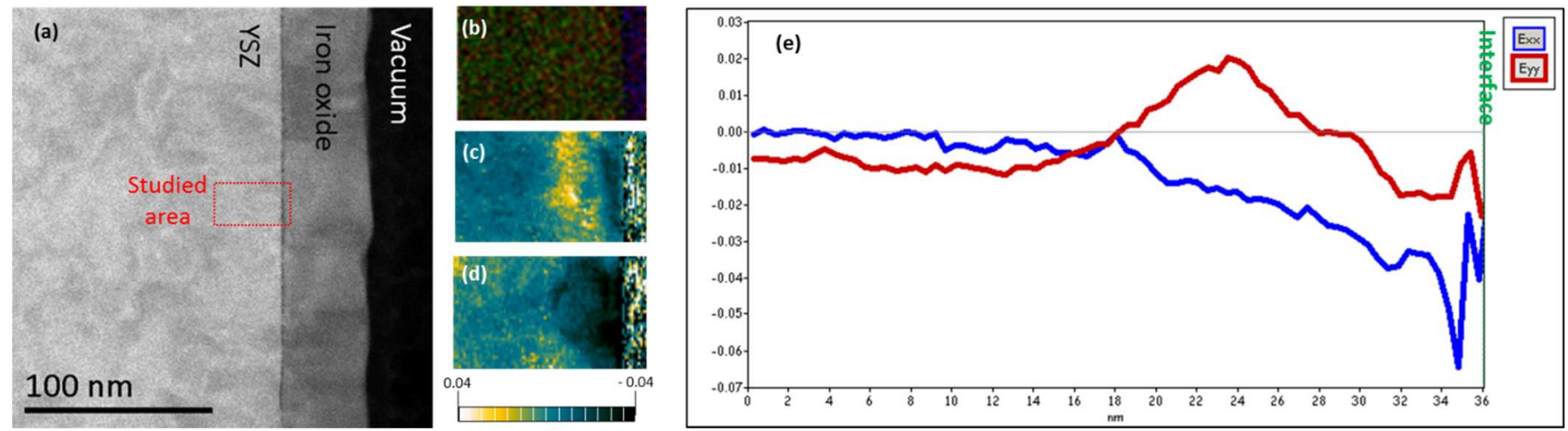

Figure 1. a) ADF STEM Survey image of $\mathrm{YSZ} / \mathrm{FeO}_{3}$ interface. The red box is the area where the electron beam was scanned for the acquisition of the simultaneous 4D STEM and EDS datasets; b) EDS maps of $\mathrm{O} \mathrm{K}$ in red, Zr L in green and Fe K in blue; c, d) in- and out-of-plane strain maps; e) profile of the strain maps (averaged over the full scanned area), the interface is labeled on the right-hand side of the graph.

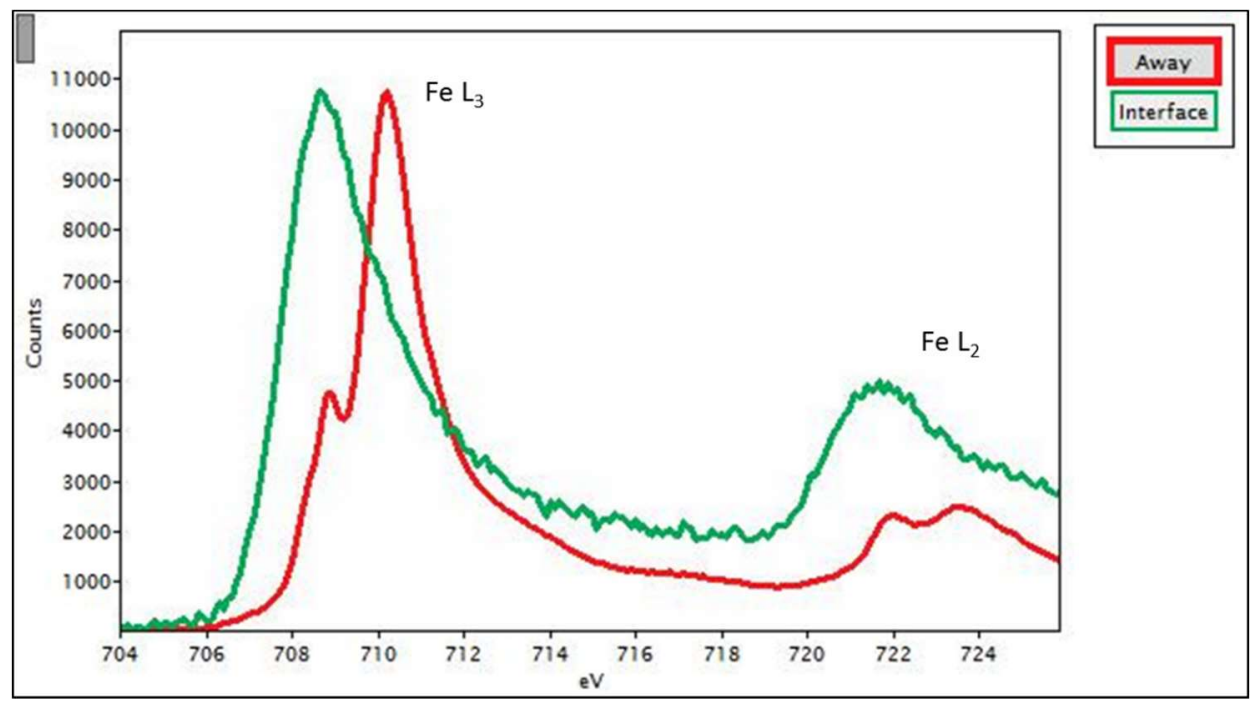

Figure 2. Fe $\mathrm{L}_{2,3}$-edges spectra extracted from the $\mathrm{Fe}_{2} \mathrm{O}_{3}$ film away from the interface with YSZ in red and right at the interface shown in green. Very significant changes in the fine structure are visible indicating variations in the chemical state of Fe. 